\title{
OPEN Early-adolescent antibiotic exposure results in mitochondrial and behavioral deficits in adult male mice
}

\author{
Anouk C. Tengeler ${ }^{1,4}$, Tim L. Emmerzaal| ${ }^{1,2,4}$, Bram Geenen ${ }^{1}$, Vivienne Verweij ${ }^{1}$, \\ Miranda van Bodegom ${ }^{1}$, Eva Morava², Amanda J. Kiliaan ${ }^{1,5}$ \& Tamas Kozicz ${ }^{1,3,5 凶}$
}

Exposure to antibiotic treatment has been associated with increased vulnerability to various psychiatric disorders. However, a research gap exists in understanding how adolescent antibiotic therapy affects behavior and cognition. Many antibiotics that target bacterial translation may also affect mitochondrial translation resulting in impaired mitochondrial function. The brain is one of the most metabolically active organs, and hence is the most vulnerable to impaired mitochondrial function. We hypothesized that exposure to antibiotics during early adolescence would directly affect brain mitochondrial function, and result in altered behavior and cognition. We administered amoxicillin, chloramphenicol, or gentamicin in the drinking water to young adolescent male wild-type mice. Next, we assayed mitochondrial oxidative phosphorylation complex activities in the cerebral cortex, performed behavioral screening and targeted mass spectrometry-based acylcarnitine profiling in the cerebral cortex. We found that mice exposed to chloramphenicol showed increased repetitive and compulsive-like behavior in the marble burying test, an accurate and sensitive assay of anxiety, concomitant with decreased mitochondrial complex IV activity. Our results suggest that only adolescent chloramphenicol exposure leads to impaired brain mitochondrial complex IV function, and could therefore be a candidate driver event for increased anxiety-like and repetitive, compulsive-like behaviors.

The brain is one of the organs that is most affected by impaired mitochondrial function, because it is one of the most metabolically active organs in the body. The brain consumes about $20 \%$ of the body's total available energy while representing only $2 \%$ of the total body weight ${ }^{1-3}$. The brain is, therefore, highly sensitive to impaired energy production ${ }^{4}$. The majority of energy used by the brain is generated by mitochondria in the form of Adenosine triphosphate (ATP). Mitochondria are also involved in brain function through the induction of apoptosis and the production of reactive oxygen species (ROS) ${ }^{4}$. Impaired mitochondrial function can, therefore, affect neurodevelopment and brain function due to the central roles of mitochondria in neurogenesis, synaptic plasticity, and neuronal activity ${ }^{1,5-8}$.

Mitochondria share many molecular and cellular characteristics with prokaryotes as a result of their common ancestry. Consequently, many antibiotics that target bacterial translation may also affect mitochondrial translation $^{9-12}$. Even at recommended treatment doses and durations, some antibiotics can damage the mitochondrial DNA, impair quality control, and disrupt energy production ${ }^{9,13}$. For example, chloramphenicol and aminoglycosides like gentamicin, bind to the prokaryotic ribosomal subunits, inhibiting protein synthesis ${ }^{14,15}$. Gentamicin binds to the small 30S prokaryotic ribosomal subunit, while chloramphenicol selectively binds to the large $50 \mathrm{~S}$ ribosomal subunit ${ }^{14,15}$.

Due to the close ribosomal similarities between prokaryotes and mitochondria, mitochondrial ribosome activity might also be affected by these antibiotics, possibly leading to inhibition of mitochondrial biogenesis ${ }^{9,16-18}$.

\footnotetext{
${ }^{1}$ Department of Medical Imaging, Anatomy, Radboud University Medical Center, Donders Institute for Brain, Cognition \& Behaviour, Centre for Medical Neuroscience, Preclinical Imaging Centre PRIME, Nijmegen, The Netherlands. ${ }^{2}$ Department of Clinical Genomics, Mayo Clinic, 200 First St. SW, Rochester, MN, USA. ${ }^{3}$ Department of Biochemistry and Molecular Biology, Mayo Clinic, Rochester, MN, USA. ${ }^{4}$ These authors contributed equally: Anouk C. Tengeler and Tim L. Emmerzaal. ${ }^{5}$ These authors jointly supervised this work: Amanda J. Kiliaan and Tamas Kozicz. ${ }^{\circledR}$ email: Kozicz.Tamas@mayo.edu
} 


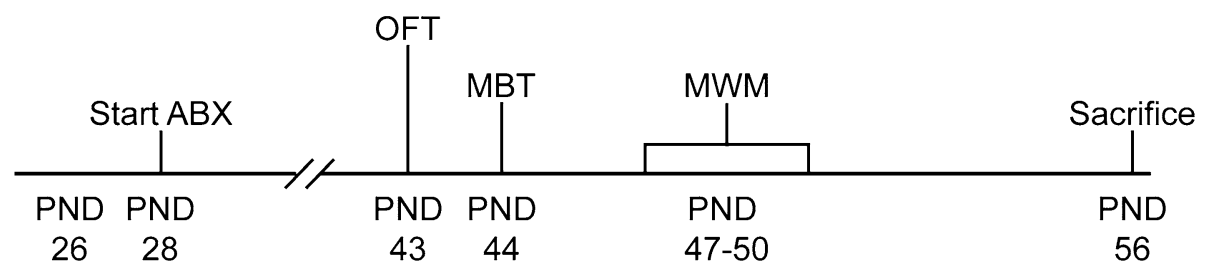

Figure 1. Timeline. Body weight and water consumption were monitored regularly, starting from postnatal day (PND) 26. From PND 28, mice received either amoxicillin, chloramphenicol, gentamicin, or no antibiotics (control group) in drinking water (start ABX). The open field test (OFT) and marble burying test (MBT) were performed on PND 43 and 44, respectively. The Morris water maze (MWM) consisted of 4 days and was assessed on PND 47-50. Mice were sacrificed via cervical dislocation on PND 56.

Dysfunctional mitochondria are reported to be involved in psychiatric disorders and symptoms such as anxiety and depression ${ }^{4,19-21}$. In addition, patients with mitochondrial DNA mutations or mitochondrial diseases often show symptoms of mood disorders ${ }^{21}$. Several classes of antibiotics that affect mitochondrial function, including aminoglycosides and fluoroquinolones, can cause neuropsychiatric adverse effects, such as mania, acute anxiety and hallucinations, in humans ${ }^{22-24}$. A nation-wide population-based Danish study by Köhler-Forsberg et al., has reported increased risks for a wide range of mental disorders, including developmental, behavioral and anxiety disorders, after infections and subsequent antibiotic exposure in childhood and adolescence ${ }^{25}$. Many of the individuals in the cohort of the study by Köhler-Forsberg et al., had severe infections, including, central nervous system (CNS) infections, urological infections, or sepsis ${ }^{25}$. These infections often require long-term treatment with antibiotics ${ }^{26,27}$.

Given the high occurrence of long-term antibiotic exposure, for example in the treatment of meningitis, osteomyelitis, and chronic urinary infection ${ }^{27-29}$, knowledge on the brain pathology following antibiotic drug exposure is of great public health relevance, however, whether such associations reflect effects of the antibiotic drug exposure remains elusive. Therefore, in this study we aimed to investigate the impact of adolescent exposure to antibiotics, like aminoglycosides and chloramphenicol, on cerebral mitochondria and behavior. Chloramphenicol is used to treat severe infections such as bacterial meningitis, osteomyelitis and typhoid fever ${ }^{30,31}$. We hypothesized that certain antibiotics could affect brain mitochondrial function possibly resulting in altered behavior and cognition. To test this hypothesis, we treated young adolescent male wild-type mice with clinically relevant doses of chloramphenicol or gentamicin in their drinking water. Because the effects could also represent general effects of the antibiotic exposure, we also treated one group of mice with amoxicillin ${ }^{32}$. Amoxicillin is part of the beta-lactam family of antibiotics and targets the bacterial cell wall synthesis ${ }^{33}$. Because mitochondria do not possess a cell wall, amoxicillin would not interfere with mitochondrial translation. Next, we assayed mitochondrial oxidative phosphorylation complex activities in the cerebral cortex, performed behavioral screening and targeted mass spectrometry-based acylcarnitine profiling in the brain cortex. We found that adolescent chloramphenicol, but not gentamicin or amoxicillin exposure, without concomitant infection(s), led to impaired brain mitochondrial complex IV function, signs of slower fatty acid metabolism, accompanied by increased anxiety-like and repetitive, compulsive-like behaviors.

\section{Materials and methods}

Mice. All animal experiments were carried out in accordance to international European ethical standards (European Directive 2010/63/EU) and they were approved by the Veterinary Authority of the Radboud university medical center (Radboudumc; Permit number: RU-DEC 2015-0077) containing a statistical power analysis to minimize group sizes. All applicable (inter)national and institutional guidelines for the care and use of animals were followed and reported in accordance with the ARRIVE guidelines ${ }^{34}$.

In total, 40 male, 28 days old C57BL/6J mice were used for this randomized and blind controlled study. The timeline of the study is illustrated in Fig. 1. Mice were group-housed with two mice in digital ventilated cages (DVC; Tecniplast S.P.A., Buguggiate (VA), Italy) and housed under standard laboratory conditions. Animals were randomly assigned to one of the four experimental groups (Control, Amoxicillin, Chloramphenicol, and Gentamicin; $\mathrm{N}=10$ per group). Body weight of all mice was measured weekly.

Antibiotic exposure. Mice were administered the following antibiotics dissolved in their drinking water as indicated: amoxicillin (0.5 g/l; A8523), chloramphenicol (0.5 g/l; C0378), or gentamicin (0.035 g/l; G1914) for 4 weeks. All antibiotics were purchased from Sigma-Aldrich, St. Louis, MO. The doses in the drinking water correspond to the approximately daily doses given to children normalized based on body weight (20-25 g) and expected water consumption $(5 \mathrm{ml} \text { per day })^{35}$. One group of mice did not receive antibiotics and served as control group. Drinking water was not sweetened to mask the taste of the antibiotics because sugar also affects body weight, behavior, and cognition. Instead, water consumption was monitored for each pair of mice by measuring water volume three times per week to prevent dehydration, and to ensure that all animals drank equal amounts of water. Drinking water of all experimental groups was refreshed three times per week.

Behavioral tests. Open Field Test (OFT) was used to evaluate locomotion and explorative behavior. Mice were placed in the center of the square open field $(45 \times 45 \times 25 \mathrm{~cm})$ with transparent Plexiglas walls, and allowed 
to freely explore the area for $10 \mathrm{~min}$. The trials were videotaped using a camera that was positioned above the open field area. Exploration activity in the center $(20 \times 20 \mathrm{~cm})$ and corners $(10 \times 10 \mathrm{~cm})$ was automatically scored and quantified using Ethovision XT10.1 (Noldus, Wageningen, The Netherlands).

We used the marble burying test (MBT) as an accurate and sensitive assay of anxiety-like, repetitive and compulsive-like behaviors in rodents ${ }^{36}$. Mice were placed in a clean conventional-sized cage preloaded with $3 \mathrm{~cm}$ clean bedding and 15 evenly spaced black glass marbles with a $14 \mathrm{~mm}$ diameter. Mice were allowed to explore the cage for $30 \mathrm{~min}$, and thereafter the cage was photographed. Three evaluators counted the number of marbles that were covered by bedding at least two-thirds of their size between trials, marbles and cages were cleaned with $70 \%$ Ethanol.

The Morris water maze (MWM) was used to assess spatial learning and memory. During the acquisition phase, the mice were trained to find the platform in a circular pool (diameter $108 \mathrm{~cm})$ that was filled with water $\left(21-22^{\circ} \mathrm{C}\right.$ ), made opaque by adding milk powder. The platform (diameter $8 \mathrm{~cm}$ ) was submerged $1 \mathrm{~cm}$ below the water surface and located in the north-east quadrant of the pool. Mice performed 4 acquisition trials per day starting from four different cardinal points (South, North, East, West; maximal swimming time $120 \mathrm{~s} ; 30 \mathrm{~s}$ on platform; inter-trial interval $60 \mathrm{~min}$ ) during 4 consecutive days. Visual cues were present on the four walls surrounding the pool at a distance of $0.5 \mathrm{~m}$. All trials were recorded and latency to find the platform (s) was used as a measure for spatial learning. Mice performed a single probe trial at the end of day 4 of acquisition, in which the platform was removed from the pool. During this probe trial, the mice were allowed to swim freely for $120 \mathrm{~s}$. Trials were recorded and analyzed with EthoVision XT 10.1 (Noldus, Wageningen, the Netherlands).

Mitochondrial analysis. Mice were sacrificed via cervical dislocation without anesthesia after which the brains were rapidly removed and dissected. The whole brain cortex was snap frozen in liquid nitrogen. A crude homogenate of the cortex was made with a glass-glass potter tube in SEF buffer (0.25 M sucrose, 2 mM K-EDTA, $10 \mathrm{mM}$ phosphate buffer, $\mathrm{pH} 7.4$ ) to obtain a $5 \% \mathrm{w} / \mathrm{v}$ homogenate. The homogenized samples were centrifuged at $600 \times g$ for $10 \mathrm{~min}$ at $2{ }^{\circ} \mathrm{C}$. The supernatant was aliquoted, snap frozen in liquid nitrogen, and stored at $-80^{\circ} \mathrm{C}$ for mitochondrial complex activity measurements.

Protein levels and enzyme activities of the individual complexes of the respiratory chain (complex I to IV), succinate: Cytochrome C oxidoreductase (SCC), and citrate synthase (CS; a marker of the number of mitochondria ${ }^{37}$ ) in the $600 \mathrm{~g}$ supernatant were measured spectrophotometrically, after three freeze-thaw cycles on a KoneLab 20XT analyzer (Thermo Scientific) following standard procedures ${ }^{38}$. These enzyme assays are based on previously described methods ${ }^{37,39-42}$. All measurements were normalized to both CS activity and protein levels.

Analysis of acylcarnitine metabolites. The whole brain cortex was also used to measure several different acylcarnitine metabolites using an UPLC-MS method as previously described ${ }^{43}$. In short, 5 mg of pulverized tissue was homogenized in $50 \mu \mathrm{l}$ of PBS before $25 \mu \mathrm{l}$ of deuterated labeled internal standards was added. Proteins were removed by adding a solution of methanol/dichloromethane $(\mathrm{v} / \mathrm{v}, 600 \mu \mathrm{l})$ to the sample mixture. The sample was centrifuged at $18,000 \times \mathrm{g}$ for $15 \mathrm{~min}$ at $4{ }^{\circ} \mathrm{C}$, and then the supernatant was transferred to a 1 dram vial, and dried under N2 stream. Samples were reconstituted and analyzed on a Waters Acquity UPLC system (Milford, MA) coupled with a Thermo Quantiva tandem mass spectrometer (West Palm Beach, FL) in positive (H) ESI mode. Concentrations of carnitine $(162.1>85.0 \mathrm{~m} / \mathrm{z})$, acetylcarnitine $(204.1>85.0 \mathrm{~m} / \mathrm{z})$, propionylcarnitine $(218.1>85.0 \mathrm{~m} / \mathrm{z})$, butyrylcarnitine $(232.1>85.0 \mathrm{~m} / \mathrm{z})$, isovalerylcarnitine $(246.1>85.0 \mathrm{~m} / \mathrm{z})$, octanoylcarnitine $(288.2>85.0 \mathrm{~m} / \mathrm{z})$, lauroylcarnitine $(344.3>85.0 \mathrm{~m} / \mathrm{z})$, myristoylcarnitine $372.3>85.0 \mathrm{~m} / \mathrm{z})$, palmitoylcarnitine $(400.4>85.0 \mathrm{~m} / \mathrm{z})$, oleoylcarnitine $(426.4>85.0 \mathrm{~m} / \mathrm{z})$, and stearoylcarnitine $(438.4>85.0 \mathrm{~m} / \mathrm{z})$ were measured against a 11-point calibration curve that underwent the same preparation.

Statistical analysis. Data were analyzed using IBM SPSS for Windows 25.0 software (SPSS Inc., Chicago, IL, USA). The latency to find the platform during the acquisition phase of the MWM, body weight, and water consumption were analyzed using repeated measures ANOVA. A MANOVA was performed for the parameters of the open field test, probe phase of the MWM, acylcarnitine, and mitochondrial complex activity measurements as dependent variables. ANOVA's were followed by Dunnett's multiple comparison test to compare the control group (mice that received water) with the treatment groups. A Kruskall-Wallis with Dunn's pairwise tests, and Bonferroni post hoc correction was used to analyze the marbles buried in the marble burying test. Statistical outliers were removed from the dataset. Significant MANOVAs and the acylcarnitine results were followed up by a discriminant function analysis (DFA). This DFA was performed with a stepwise approach and Mahalanobis distance as method.

\section{Results}

Administration of antibiotics in drinking water had no effects on water consumption or body weight. Body weight and water consumption were measured to evaluate the effects of antibiotics on these parameters. Initial body weight did not differ between the experimental groups, and all mice gained body weight over the next 4 weeks $[\mathrm{F}(4,144)=431.65, \mathrm{p}<0.001$; Fig. 2a]. No differences in body weight gain between the experimental groups were detected.

The pairs of mice consumed $13.8 \pm 0.3 \mathrm{~mL}$ water daily before start of the antibiotics administration. No differences in water consumption from week 0 to week 4 were observed. Water consumption was comparable between the experimental groups (Fig. 2b).

Chloramphenicol treatment affects repetitive and anxiety-related behavior in mice. We examined locomotion, explorative behavior, spatial memory and spatial learning in the OFT and MWM. We 
a

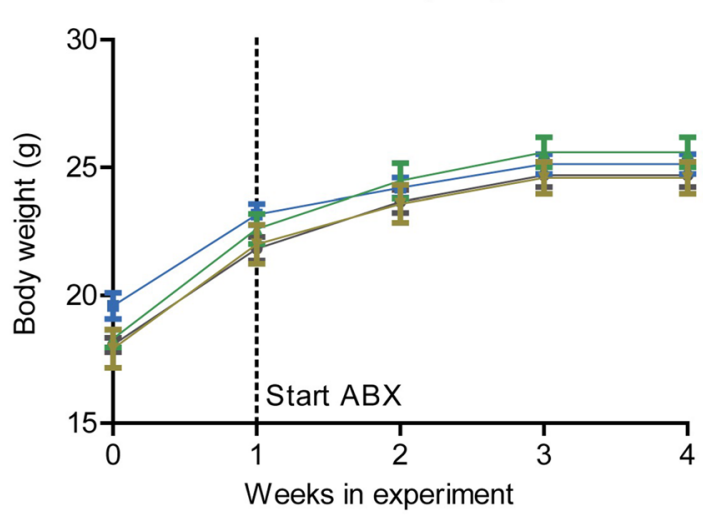

b

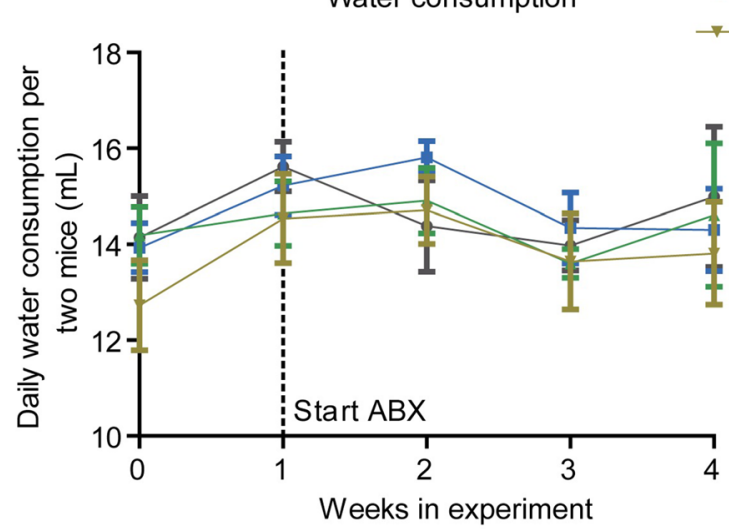

Figure 2. Body weight and water consumption. The body weight of all mice was measured weekly before start of antibiotics administration (week 0$)$ and after (week 1-4). Mice gained weight over the weeks $(\mathrm{p}<0.001)$. No differences between the experimental groups were observed (A). Daily water consumption per two mice before treatment with antibiotics (week 0 ) and after (week 1-4). No differences between the groups were detected (B). CAP, chloramphenicol. $\mathrm{N}=10$ per group. Data are presented as mean $\pm \mathrm{SEM}$.

found no differences in locomotion and explorative behavior in the OFT (Fig. 3a,b). Spatial learning and memory of the mice were tested with the MWM test. All mice learned to find the hidden platform during the acquisition phase, and this was not affected by any of the treatments (Fig. 3c). During the probe phase of the MWM, the platform was removed. No differences between the groups were observed in the latency to go to the former platform location (Fig. 3d), even after controlling for mean swimming velocity. No differences in mean swimming velocity (Fig. 3e) and the total swimming distance (Fig. 3f) were observed as well. These data suggest that treating rodents with either amoxicillin, chloramphenicol or gentamicin does not influence overall activity, exploration and spatial learning and memory.

An accurate and sensitive assay of anxiety-like, repetitive and compulsive-like behaviors in mice was performed by using the marble burying test ${ }^{36}$. Marble burying behavior was significantly affected by antibiotic administration $(\mathrm{H}(3)=9.76, \mathrm{p}<0.05$; Fig. 3g). Dunn's pairwise tests revealed a significant increase in marbles buried by mice treated with chloramphenicol compared to the control group $(\mathrm{p}=0.019)$. Gentamicin and amoxicillin did not affect marble burying behavior.

Chloramphenicol treatment results in decreased activity of the respiratory chain complex IV. Mitochondrial complex activity was measured in the cortex of all mice. One control mouse was considered a statistical outlier and was removed from further analyses. All measurements were normalized against CS activity per mg protein which did not differ between the experimental groups (Fig. 4a). The activity of respiratory chain complexes I, II, and III, and SCC activity were not affected by the studied antibiotics (Fig. 4b-e). However, cytochrome $\mathrm{C}$ oxidase (COX), which was used to measure complex IV activity, showed a reduced activity in mice treated with chloramphenicol compared to vehicle treated mice $(p=0.035$; Fig. $4 \mathrm{f})$. These results reveal that chloramphenicol, besides its effect on behavior, reduces brain mitochondrial complex IV function.

The MANOVA was followed up with a discriminant function analysis (DFA) to assess whether groups differ along a linear combination of outcome variables. Predictors were activity of mitochondrial complex IV, and number of marbles buried in the MBT. The DFA revealed that activity of mitochondrial complex IV was the main predictor that explained $100 \%$ of variance, canonical $\mathrm{R}^{2}=0.457$. This discriminant function significantly differentiated the antibiotics groups, $\Lambda=0.791, \chi^{2}(3)=8.323, \mathrm{p}=0.04$.

The classification results showed that $25.6 \%$ of all cases were correctly classified; $40 \%$ of mice treated with chloramphenicol were classified correctly, whereas $11.1 \%$ of control mice were correctly predicted. Of amoxicillin-treated and gentamicin-treated mice, $0 \%$ and $20 \%$ were correctly classified, respectively.

Antibiotic treatment alters carnitine metabolism. Acylcarnitine metabolism is a classical marker of mitochondrial dysfunction. Therefore, next we explored whether acylcarnitine metabolism is altered in chloramphenicol exposed mice with decreased COX function. We assessed free carnitine (C0) and 11 acylcarnitine species in control, chloramphenicol, and gentamicin treated mice. All results were corrected to total carnitine concentrations to normalize the data (Fig. 5a). We found significant differences between treatment groups in free carnitine $(C 0 ; p=0.033)$, acetylcarnitine $(C 2 ; p=0.031)$, and isobutyrylcarnitine (Iso-C4; $p=0.042)$. Post hoc multiple comparisons revealed that gentamicin treatment increased $\mathrm{C} 0(\mathrm{p}=0.032$; Fig. $5 \mathrm{~b})$ concentrations, while chloramphenicol treatment only tended to increase $\mathrm{C} 0$ abundances $(\mathrm{p}=0.059)$. Conversely, post hoc comparisons revealed that gentamicin treatment negatively affected C2 concentrations $(p=0.030$; Fig. $5 c)$, while chloramphenicol only tended to decrease $\mathrm{C} 2$ abundance $(\mathrm{p}=0.056)$. Although Iso-C4 concentrations varied 
a

OFT velocity

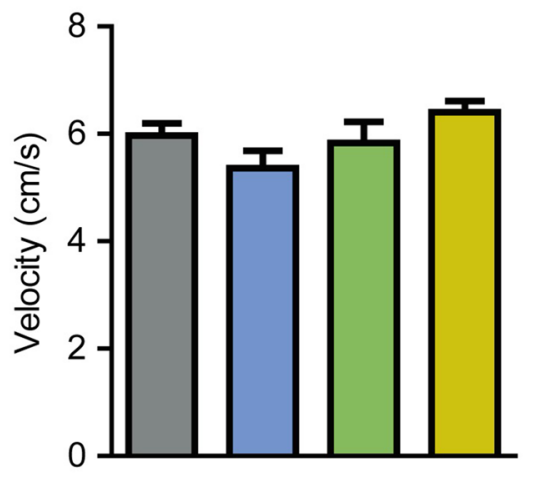

b

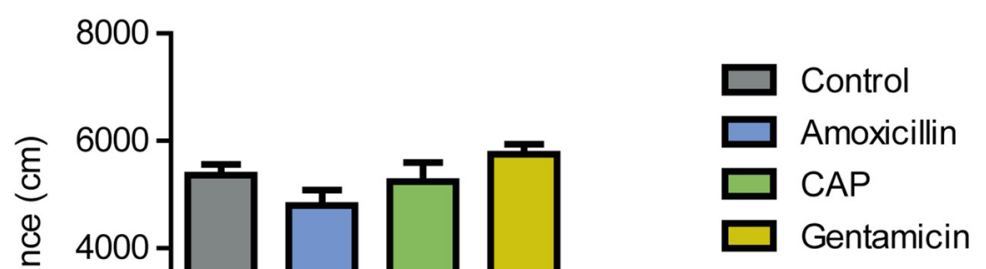

C

MWM acquisition

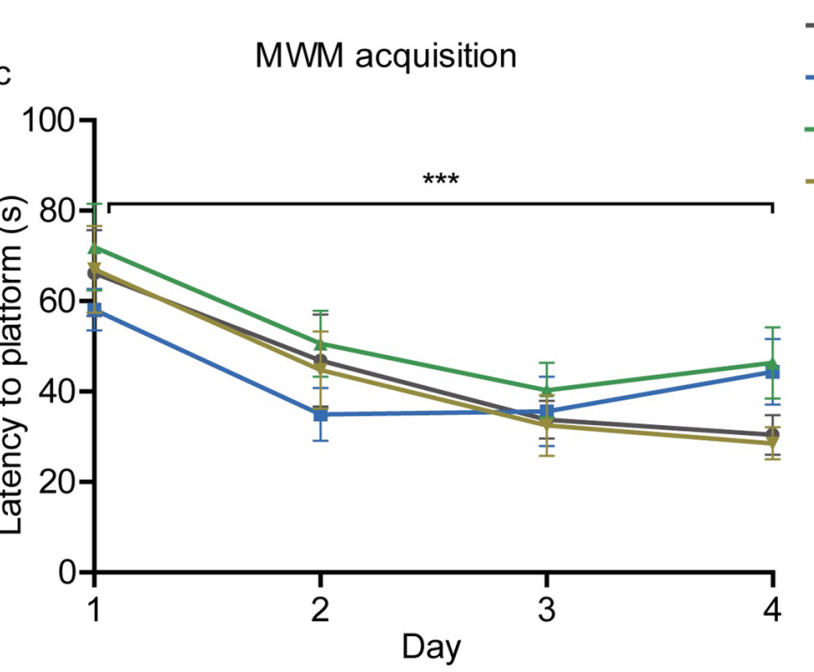

$\rightarrow$ Control

$\because$ Amoxicillin

d

MWM probe latency

$\simeq$ CAP

$\div$ Gentamicin

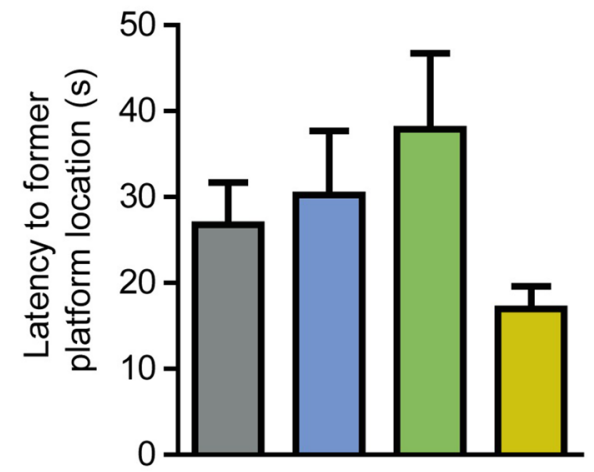

e

f

MWM probe distance

g Marble burying test
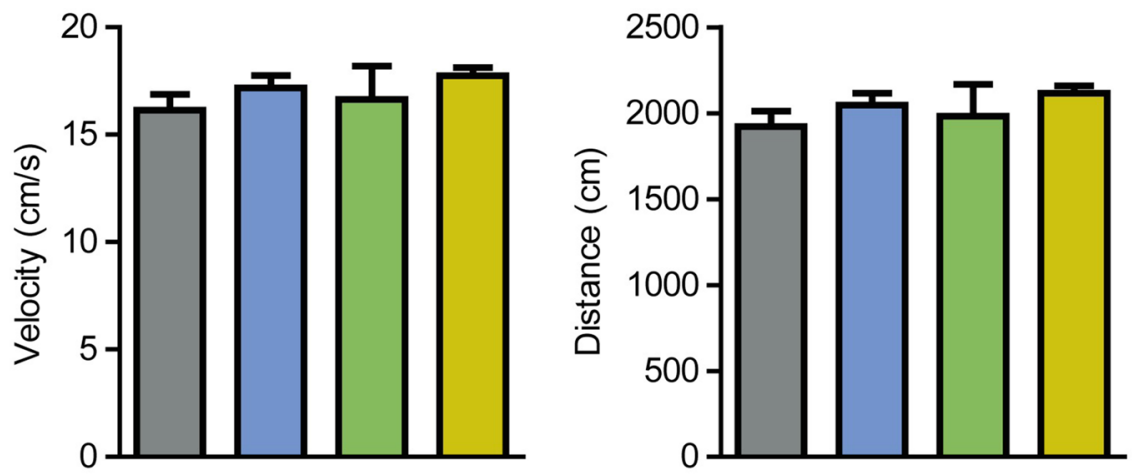

Figure 3. Behavior and cognition assessed with the OFT, MWM and MBT. Mean walking velocity (A) and total distance walked (B) during the OFT. No differences between the experimental groups were detected. Spatial learning assessed with the MWM in a 4-day acquisition phase. All groups showed a learning curve, but no differences between the groups were observed $(\mathbf{C})$. The latency to go to the former platform location in the probe phase of the MWM (D), and the mean swimming velocity (E) and total swimming distance (F) were not affected by the antibiotics assessed. Buried marbles (i.e., more than two-thirds of its surface area) were scored by 3 task scorers $(\mathbf{G})$. The scores for each mouse were averaged for all scorers. Mice that were administered chloramphenicol buried more marbles than control mice $(\mathrm{p}=0.019)$. CAP, chloramphenicol. $\mathrm{N}=10$ per group. Data are presented as mean \pm SEM. 
a

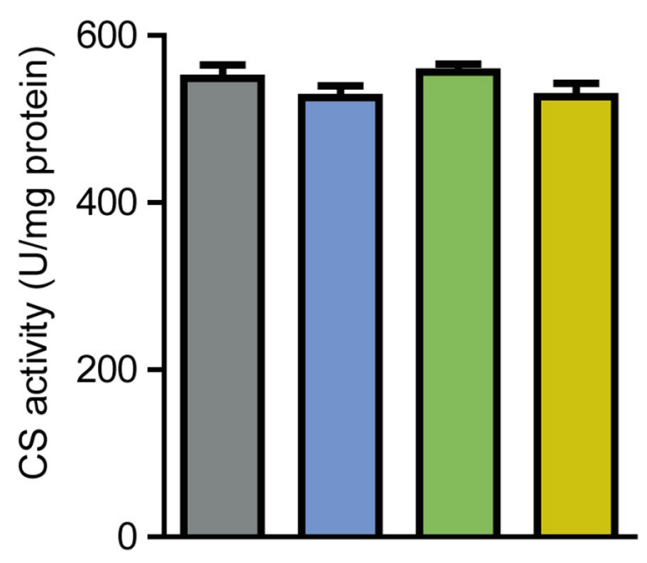

C

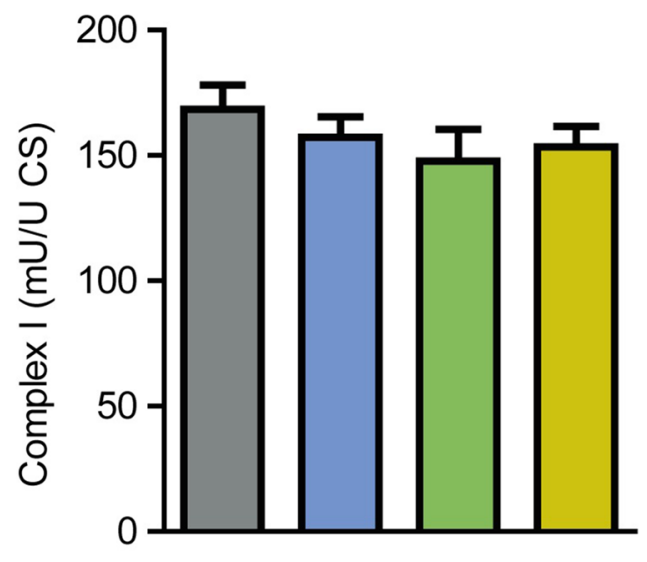

e

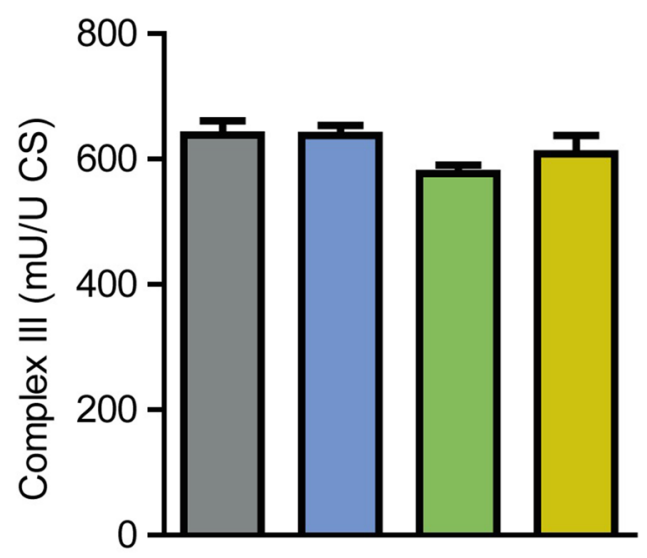

b

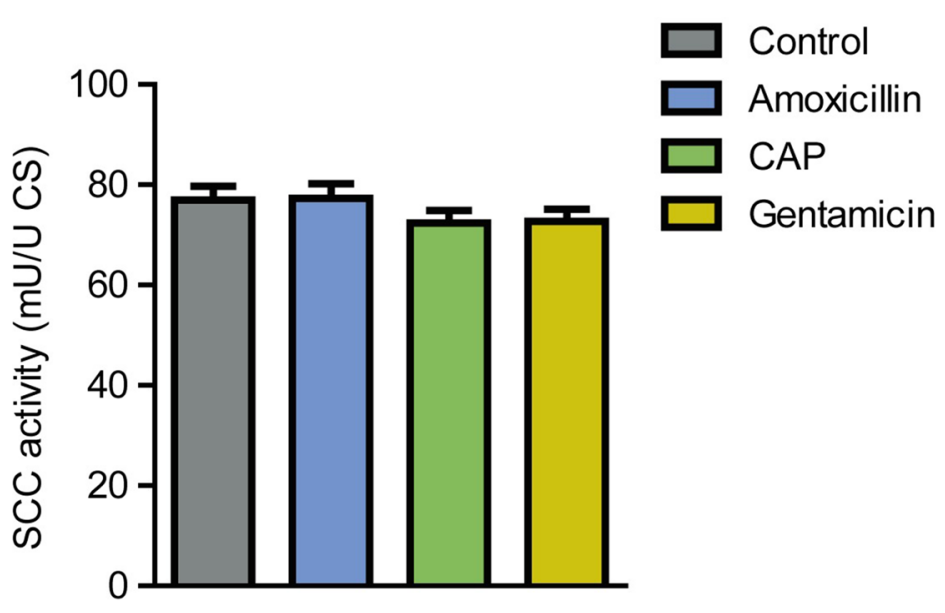

d

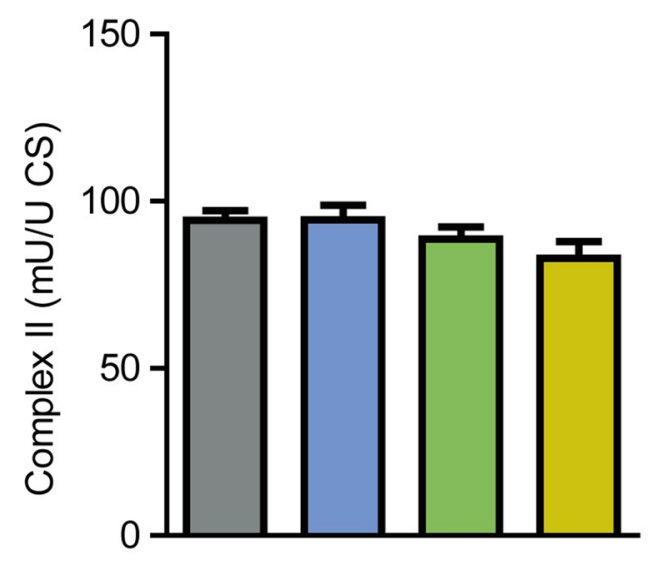

f

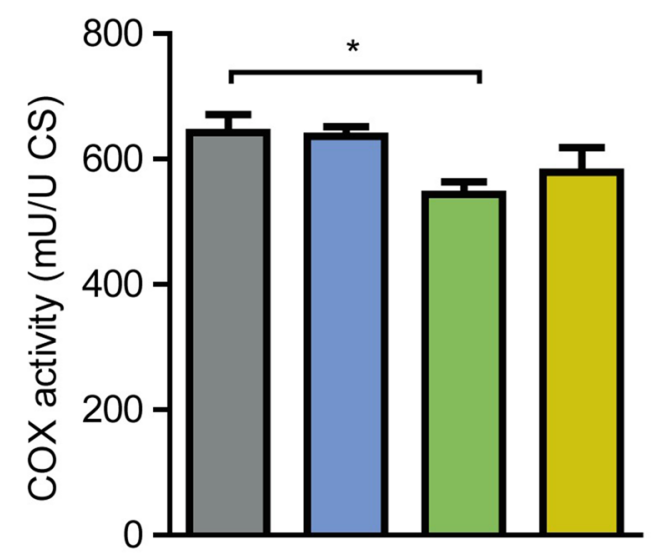

Figure 4. Activity of mitochondrial complexes. CS activity was used to normalize all measurements. CS activity did not differ between the groups (A). SCC activity (B), Complex I activity (C), complex II activity (D), complex III activity (E), and COX activity (F) were measured in all mice. COX activity, used to measure complex IV activity, was decreased in mice that were administered chloramphenicol $(\mathrm{p}=0.035)$. CAP, chloramphenicol; CS, citrate synthase; $\mathrm{COX}$, cytochrome c oxidase. $\mathrm{N}=9$ (control) and $\mathrm{N}=10$ (all other groups). Data are presented as mean \pm SEM. 


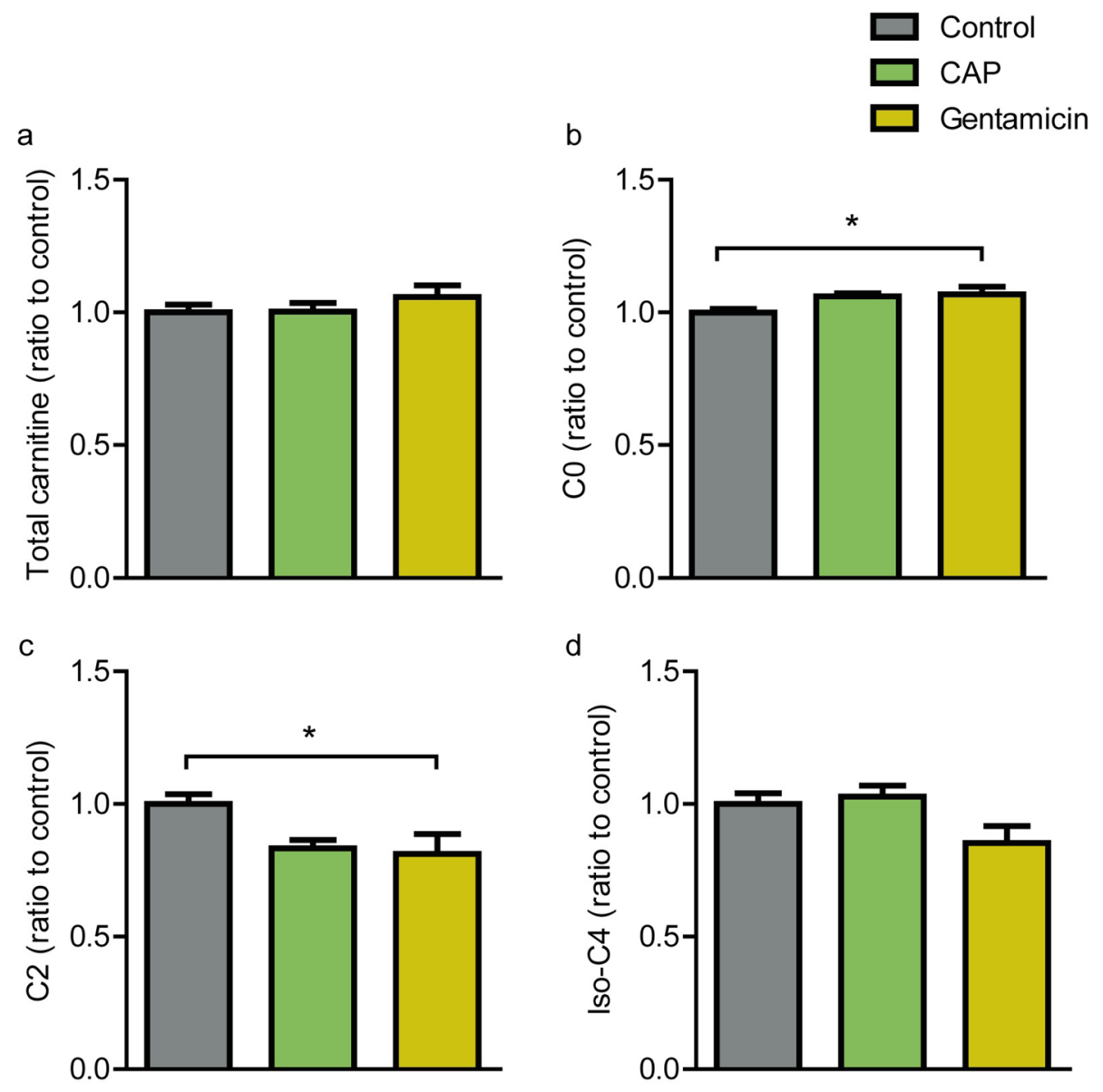

Figure 5. Acylcarnitine metabolite profile. Total carnitine concentration (A) was used to normalize all individual acylcarnitine measurements. Ratio of free carnitine (B), Acetylcarnitine (C), and isobutyrylcarnitine (D). CAP, chloramphenicol. $\mathrm{N}=10$ mice per group. Data are presented as mean $\pm \mathrm{SEM}$.

significantly between the different treatments, this was not influenced by antibiotic treatment compared to the control group (Fig. 5d). These results identify brain acylcarnitine metabolism derangements in mice treated with gentamicin.

These results were followed up by a stepwise DFA that showed that both Iso-C4 and C0 were the main predictors for differentiation between the groups. The two discriminant functions explained 53.7\% (canonical $\mathrm{R}^{2}=0.474$ ) and $46.3 \%$ (canonical $\mathrm{R}^{2}=0.447$ ) of the variance, respectively. Combining these discriminant functions show that they significantly differentiate the groups, $\Lambda=0.621, \chi^{2}(4)=12.625, p=0.013$ (Fig. 6).

\section{Discussion}

Despite the high occurrence of exposure to antibiotics during adolescence and its association with increased risk for developing psychopathology ${ }^{25,44,45}$, an important research gap exists in understanding how antibiotic therapy during adolescence could lead to increased vulnerability to developing psychopathology. Finding potential driver events explaining this association remains of public health relevance.

Here we show that adolescent exposure to chloramphenicol but not to amoxicillin or gentamicin is associated with decreased mitochondrial complex IV activity in the brain, and with increased anxiety-like, repetitive and compulsive-like behaviors in male mice. Stepwise DFA confirmed that mice exposed to chloramphenicol could clearly be discriminated from the other experimental groups using one predictor: activity of mitochondrial complex IV. Of mice that were treated with chloramphenicol, $70 \%$ could be classified correctly.

Complex IV is a highly regulated enzyme that is involved in the reduction of $\mathrm{O}_{2}$ to $\mathrm{H}_{2} \mathrm{O}^{46}$. As the final electron carrier in the electron transport chain, complex IV acts as the rate-limiting step for electron transfer. Complex IV activity is therefore an indication of the oxidative capacity of the cells ${ }^{47}$. Mitochondrial complex I, II and III were not affected in this study. This has also been reported in a study in which the effects of chloramphenicol on bovine aortic endothelial cells were explored. In that study, chloramphenicol also inhibited mitochondrial protein synthesis resulting in a decrease of complex IV activity ${ }^{48}$. Besides that, they also found no effects of chloramphenicol on complex II or complex III. Another in vitro study showed that chloramphenicol lowered the expression of the mitochondrial DNA (mtDNA)-encoded COX 1 subunit of complex IV, resulting in a reduction of COX1 protein levels ${ }^{49}$. Chloramphenicol, and other antibiotics that target ribosome function, inhibits 


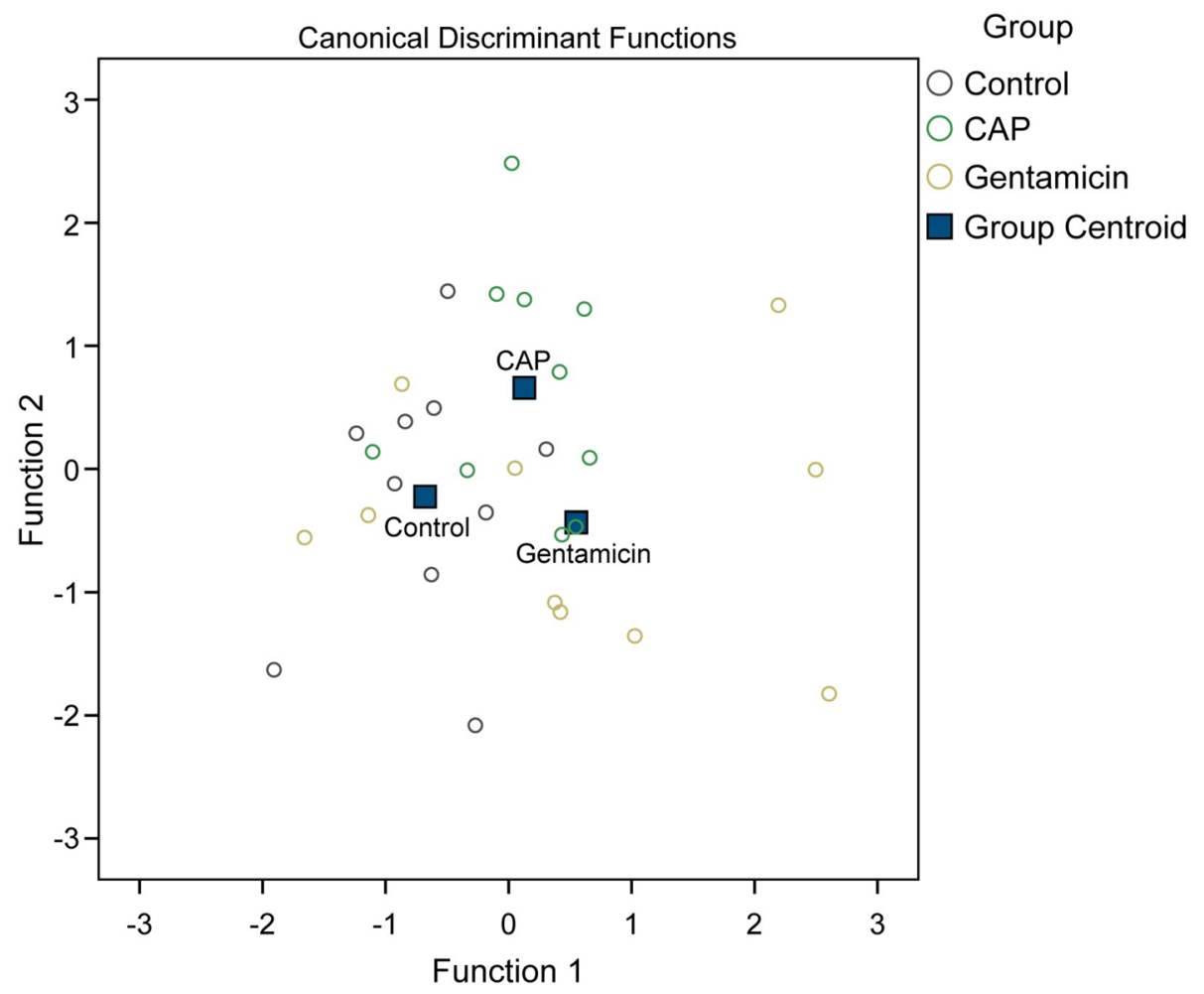

Figure 6. Discriminant function analysis. Canonical variables plot of the discriminant function analysis that classifies control (blue circles), chloramphenicol (green circles) and gentamicin (yellow circles) samples based on the acylcarnitine profile. $\mathrm{N}=10$ per group.

translation of protein encoded by the mitochondrial, but not the nuclear, genome ${ }^{32}$. A relative imbalance between the expression of nuclear and mitochondrially encoded proteins of the subunits of the mitochondrial complexes, termed mitonuclear protein imbalance, could drive mitochondrial complex instability ${ }^{50,51}$, possible resulting in decreased complex IV activity. However, the exact mechanisms through which chloramphenicol impacts complex IV activity in vivo remains to be explored in future studies.

We did not detect an effect of gentamicin on mitochondrial function in the brain cortex of the mice. It is possible that this is due to differences in permeability across the blood-brain barrier. Whereas chloramphenicol readily crosses the blood-brain barrier, the permeability of this barrier to gentamicin and other aminoglycosides is shown to be poor ${ }^{52}$. Finally, administration of amoxicillin did not affect brain mitochondrial functions either. This is consistent with its mode of action in bacteria since it disrupts the bacterial cell wall and therefore would not affect mitochondrial function.

The brain has the highest mitochondrial energy demand of any organ. Multiple pieces of preclinical and clinical evidence suggest that brain mitochondrial function is impaired in the pathobiology of neuropsychiatric presentations ${ }^{1,53-56}$. Our results therefore suggest that adolescent chloramphenicol exposure alone, without infection(s) leads to impaired brain mitochondrial complex IV function that could be a candidate driver event for increased anxiety, repetitive and compulsive-like behaviors, although the causality of this relationship should be investigated in future studies.

Carnitine, that conjugates with acyl-coenzyme A (acyl-CoA) to form acylcarnitine, is vital for transport of fatty acids into mitochondria for subsequent $\beta$-oxidation resulting in acetyl-CoA and electron carriers that deliver electrons to the electron transport chain ${ }^{57,58}$. Altered plasma concentrations of acylcarnitines are suggested markers of impaired metabolism, although acylcarnitine profiles are usually nonspecific ${ }^{59}$. Acetyl-CoA can be converted to acetylcarnitine, which is the shortest acylcarnitine. Acetylcarnitine can cross the mitochondrial membrane and enables mitochondrial efflux of excess acyl groups. We found that acetylcamitine (C2) was decreased during gentamicin treatment. This was accompanied by an increase in free-carnitine levels as a possible consequence of decrease in acyl group transfer and metabolism.

Chloramphenicol, an antibiotic which is able to cross the blood-brain barrier, affected mitochondrial complex IV activity, whereas gentamicin, which cannot easily pass the blood-brain barrier, only impacted the acylcarnitine metabolites profile, but not complex IV activity in the brain. This could suggest that chloramphenicol directly affects cerebral mitochondria, while the effects of gentamicin are indirect, for example via the gut microbiota. It has been extensively discussed in the literature that the use of both single and multiple antibiotic regimens change the gut microbiota and affects the brain-gut axis ${ }^{60-62}$. In this study we did not assess the microbiota, therefore, whether antibiotic exposure directly alters mitochondrial function and the animal's behavior, or indirectly, for example via altering the gut microbiome, remains to be explored in future studies. 
When interpreting our results, one should also consider that chloramphenicol is capable of inducing oxidative stress. Although not measured in this study, high levels of reactive oxygen species (ROS) and nitric oxide (NO), for instance in case of mitochondrial complex IV dysfunction, are also associated with high anxiety and compulsive behavior in mice ${ }^{63,64}$.

This study is not without technical considerations. Serial oral gavage is the most often used method to administer precise doses of antibiotics to mice. This route of administration is also associated with adverse effects like stress ${ }^{13}$ that could be a potential confound in the outcome of behavioral tests. We chose to administer antibiotics via unsweetened drinking water to mice. Although this could be seen as a potential limitation (animals consume less water), our results show that administration of antibiotics via unsweetened drinking water did not alter water consumption. This study only used male mice. Several psychopathologies have been associated with early antibiotics treatment, such as attention deficit hyperactivity disorder (ADHD) and autism spectrum disorder (ASD). These disorders are more prevalent in males than females, although this may be a consequence of diagnostic bias, due to differences in symptom presentation ${ }^{65,66}$. It is, however, of utmost importance to include female mice in future studies to delineate the impact of antibiotic treatment on brain pathology. As dysfunctional mitochondria are reported to be involved in psychiatric disorders like anxiety disorders and depression ${ }^{4,19-21}$, it would be worthwhile to include behavioral tests to explore depression-like behavior as well in further studies. Future studies should also evaluate effects of the antibiotics at a later time point, to differentiate between immediate and long-lasting effects of the antibiotics exposure. Also, in this study we investigated mitochondrial function in the cerebral cortex of the mice. Future research may consider to explore mitochondrial function in other brain regions as well. Additionally, mitochondrial function in other tissues, for instance muscle or liver, might be worthwhile to study as well, because peripheral effects could be partially responsible for the effects of chloramphenicol on behavior.

Exposure to infections and concomitant antibiotic treatment are associated with increased vulnerability to develop various complex neuropsychiatric disorders. However, whether such increased risk for developing neuropsychopathology reflects effects of the antibiotic drug exposure, that of the infections or both, remains incompletely defined. In this study, we focused on the effects of antibiotic treatment in the absence of infections. The link between infections and complex neuropsychiatric disorders, and the potential modulating role of antibiotics, remains to be studied in future research.

In conclusion, we present evidence that adolescent exposure to chloramphenicol leads to bioenergetic scars that may be implicated in the risk for developing psychopathology. The collateral damage of adolescent antibiotic exposure could be long lasting. More knowledge on the impact of antibiotics exposure on mitochondrial function could help us develop strategies to reduce the impact of adolescent antibiotics exposure on the brain.

\section{Data availability}

The datasets generated during and/or analyzed during the current study are available from the corresponding author on reasonable request.

Received: 10 February 2021; Accepted: 21 May 2021

Published online: 18 June 2021

\section{References}

1. Morava, E. \& Kozicz, T. Mitochondria and the economy of stress (mal)adaptation. Neurosci. Biobehav. Rev. 37, 668-680 (2013).

2. Wang, Z., Zhang, J., Ying, Z. \& Heymsfield, S. B. Organ-tissue level model of resting energy expenditure across mammals: New insights into Kleiber's law. ISRN Zool. 2012, 9. https://doi.org/10.5402/2012/673050 (2012).

3. Kummitha, C. M., Kalhan, S. C., Saidel, G. M. \& Lai, N. Relating tissue/organ energy expenditure to metabolic fluxes in mouse and human: Experimental data integrated with mathematical modeling. Physiol. Rep. https://doi.org/10.14814/phy2.12159 (2014).

4. Allen, J., Romay-Tallon, R., Brymer, K. J., Caruncho, H. J. \& Kalynchuk, L. E. Mitochondria and mood: Mitochondrial dysfunction as a key player in the manifestation of depression. Front. Neurosci. 12, 386 (2018).

5. Ames, A. CNS energy metabolism as related to function. Brain Res. Rev. 34, 42-68 (2000).

6. Erecinska, M., Cherian, S. \& Silver, I. A. Energy metabolism in mammalian brain during development. Prog. Neurobiol. 73, $397-445$. https://doi.org/10.1016/j.pneurobio.2004.06.003 (2004).

7. Kann, O. \& Kovacs, R. Mitochondria and neuronal activity. Am. J. Physiol. Cell Physiol. 292, C641-657. https://doi.org/10.1152/ ajpcell.00222.2006 (2007).

8. Emmerzaal, T. L. et al. Impaired mitochondrial complex I function as a candidate driver in the biological stress response and a concomitant stress-induced brain metabolic reprogramming in male mice. Transl. Psychiatry 10, 176 (2020).

9. Kalghatgi, S. et al. Bactericidal antibiotics induce mitochondrial dysfunction and oxidative damage in Mammalian cells. Sci. Transl. Med. 5, 185-192. https://doi.org/10.1126/scitranslmed.3006055 (2013).

10. Duewelhenke, N., Krut, O. \& Eysel, P. Influence on mitochondria and cytotoxicity of different antibiotics administered in high concentrations on primary human osteoblasts and cell lines. Antimicrob. Agents Chemother. 51, 54-63. https://doi.org/10.1128/ AAC.00729-05 (2007).

11. Li, Q., Peng, S., Sheng, Z. \& Wang, Y. Ofloxacin induces oxidative damage to joint chondrocytes of juvenile rabbits: excessive production of reactive oxygen species, lipid peroxidation and DNA damage. Eur. J. Pharmacol. 626, 146-153. https://doi.org/10. 1016/j.ejphar.2009.09.044 (2010).

12. Ghaly, H., Jorns, A. \& Rustenbeck, I. Effect of fluoroquinolones on mitochondrial function in pancreatic beta cells. Eur. J. Pharm. Sci. 52, 206-214. https://doi.org/10.1016/j.ejps.2013.11.011 (2014).

13. Wang, X., Ryu, D., Houtkooper, R. H. \& Auwerx, J. Antibiotic use and abuse: A threat to mitochondria and chloroplasts with impact on research, health, and environment. BioEssays 37, 1045-1053 (2015).

14. Wilson, D. N. On the specificity of antibiotics targeting the large ribosomal subunit. Ann. N. Y. Acad. Sci. 1241, 1-16 (2011).

15. Sutcliffe, J. A. Improving on nature: Antibiotics that target the ribosome. Curr. Opin. Microbiol. 8, 534-542 (2005).

16. Li, C. H., Cheng, Y. W., Liao, P. L., Yang, Y. T. \& Kang, J. J. Chloramphenicol causes mitochondrial stress, decreases ATP biosynthesis, induces matrix metalloproteinase-13 expression, and solid-tumor cell invasion. Toxicol. Sci. 116, 140-150. https://doi.org/ $10.1093 /$ toxsci/kfq085 (2010). 
17. Hirose, K., Hockenbery, D. M. \& Rubel, E. W. Reactive oxygen species in chick hair cells after gentamicin exposure in vitro. Hear. Res. 104, 1-14 (1997).

18. Oishi, N. et al. XBP1 mitigates aminoglycoside-induced endoplasmic reticulum stress and neuronal cell death. Cell Death Dis. 6, e1763. https://doi.org/10.1038/cddis.2015.108 (2015).

19. Flippo, K. H. \& Strack, S. An emerging role for mitochondrial dynamics in schizophrenia. Schizophr. Res. 187, $26-32$ (2017).

20. Rezin, G. T., Amboni, G., Zugno, A. I., Quevedo, J. \& Streck, E. L. Mitochondrial dysfunction and psychiatric disorders. Neurochem. Res. 34, 1021-1029 (2009).

21. Morava, E. et al. Depressive behaviour in children diagnosed with a mitochondrial disorder. Mitochondrion 10, 528-533 (2010).

22. Kandasamy, A. \& Srinath, D. Levofloxacin-induced acute anxiety and insomnia. J. Neurosci. Rural Pract. 3, 212-214 (2012).

23. Stefano, G. B., Samuel, J. \& Kream, R. M. Antibiotics may trigger mitochondrial dysfunction inducing psychiatric disorders. Med. Sci. Monit. 23, 101-106 (2017).

24. Lambrichts, S., Van Oudenhove, L. \& Sienaert, P. Antibiotics and mania: A systematic review. J. Affect. Disord. 219, 149-156 (2017).

25. Kohler-Forsberg, O. et al. A nationwide study in Denmark of the association between treated infections and the subsequent risk of treated mental disorders in children and adolescents. JAMA Psychiat. 76, 271-279 (2019).

26. Hoffman, O. \& Weber, R. J. Pathophysiology and treatment of bacterial meningitis. Ther. Adv. Neurol. Disord. 2, 1-7 (2009).

27. Le Saux, N. \& Canadian Paediatric Society, I. D. \& Immunization, C. Guidelines for the management of suspected and confirmed bacterial meningitis in Canadian children older than one month of age. Paediatr. Child. Health 19, 141-152 (2014).

28. Molyneux, E. M., Dube, Q. \& Newberry, L. Improving the outcome of bacterial meningitis in newborn infants in Africa: Reflections on recent progress. Curr. Opin. Infect. Dis. 28, 215-220 (2015).

29. Kang, C. I. et al. Clinical practice guidelines for the antibiotic treatment of community-acquired urinary tract infections. Infect. Chemother. 50, 67-100 (2018).

30. Nadel, S. Treatment of meningococcal disease. J. Adolesc. Health. 59, S21-28. https://doi.org/10.1016/j.jadohealth.2016.04.013 (2016).

31. Eliakim-Raz, N. et al. Efficacy and safety of chloramphenicol: Joining the revival of old antibiotics? Systematic review and metaanalysis of randomized controlled trials. J. Antimicrob. Chemother. 70, 979-996. https://doi.org/10.1093/jac/dku530 (2015).

32. Moullan, N. et al. Tetracyclines disturb mitochondrial function across eukaryotic models: A call for caution in biomedical research. Cell. Rep. 10, 1681-1691 (2015).

33. AlNeyadi, S. S., Salem, A. A., Ghattas, M. A., Atatreh, N. \& Abdou, I. M. Antibacterial activity and mechanism of action of the benzazole acrylonitrile-based compounds: Invitro, spectroscopic, and docking studies. Eur. J. Med. Chem. 136, 270-282 (2017).

34. Kilkenny, C., Browne, W. J., Cuthill, I. C., Emerson, M. \& Altman, D. G. Improving bioscience research reporting: The ARRIVE guidelines for reporting animal research. PLoS Biol. 8, e1000412. https://doi.org/10.1371/journal.pbio.1000412 (2010).

35. Sharland, M., Cant, A. \& Singadia, D. Manual of childhood infections: the blue book. 3rd edn, 815, 826, 836 (Oxford University Press, 2011).

36. Angoa-Perez, M., Kane, M. J., Briggs, D. I., Francescutti, D. M. \& Kuhn, D. M. Marble burying and nestlet shredding as tests of repetitive, compulsive-like behaviors in mice. J. Vis. Exp. 82, 50978 (2013).

37. Srere, P. Citrate synthase:[EC 4.1 3.7 Citrate oxaloacetate-lyase (CoA-acetylating)]. Methods Enzymol. 13, 3-11 (1969).

38. Rodenburg, R. J. Biochemical diagnosis of mitochondrial disorders. J. Inherit. Metab. Dis. 34, 283-292 (2011).

39. Cooperstein, S. \& Lazarow, A. A microspectrophotometric method for the determination of cytochrome oxidase. J. Biol. Chem. 189, 665-670 (1951).

40. Janssen, A. J. et al. Spectrophotometric assay for complex I of the respiratory chain in tissue samples and cultured fibroblasts. Clin. Chem. 53, 729-734 (2007).

41. Mourmans, J. et al. Clinical heterogeneity in respiratory chain complex III deficiency in childhood. J. Neurol. Sci. 149, 111-117 (1997).

42. Fischer, J. C. et al. Differential investigation of the capacity of succinate oxidation in human skeletal muscle. Clin. Chim. Acta 153, 23-36 (1985).

43. Chace, D. H. et al. Electrospray tandem mass spectrometry for analysis of acylcarnitines in dried postmortem blood specimens collected at autopsy from infants with unexplained cause of death. Clin. Chem. 47, 1166-1182 (2001).

44. Lavebratt, C. et al. Early exposure to antibiotic drugs and risk for psychiatric disorders: a population-based study. Transl. Psychiatry 9, 317. https://doi.org/10.1038/s41398-019-0653-9 (2019).

45. Al-Haddad, B. J. S. et al. The fetal origins of mental illness. Am J Obstet Gynecol 221, 549-562 (2019).

46. Bourens, M., Fontanesi, F., Soto, I. C., Liu, J. \& Barrientos, A. Redox and reactive oxygen species regulation of mitochondrial cytochrome C oxidase biogenesis. Antioxid. Redox Signal 19, 1940-1952 (2013).

47. Srinivasan, S. \& Avadhani, N. G. Cytochrome c oxidase dysfunction in oxidative stress. Free Radic. Biol. Med. 53, 1252-1263 (2012).

48. Ramachandran, A. et al. Inhibition of mitochondrial protein synthesis results in increased endothelial cell susceptibility to nitric oxide-induced apoptosis. Proc. Natl. Acad. Sci. USA 99, 6643-6648. https://doi.org/10.1073/pnas.102019899 (2002).

49. Santo-Domingo, J., Chareyron, I., Broenimann, C., Lassueur, S. \& Wiederkehr, A. Antibiotics induce mitonuclear protein imbalance but fail to inhibit respiration and nutrient activation in pancreatic beta-cells. Exp. Cell. Res. 357, 170-180 (2017).

50. Fuentes-Retamal, S. et al. Complex mitochondrial dysfunction induced by TPP+-gentisic acid and mitochondrial translation inhibition by doxycycline evokes synergistic lethality in breast cancer cells. Cells $\mathbf{9 , 4 0 7}$ (2020).

51. Houtkooper, R. H. et al. Mitonuclear protein imbalance as a conserved longevity mechanism. Nature 497, 451-457 (2013).

52. Sullins, A. K. \& Abdel-Rahman, S. M. Pharmacokinetics of antibacterial agents in the CSF of children and adolescents. Paediatr. Drugs 15, 93-117. https://doi.org/10.1007/s40272-013-0017-5 (2013).

53. Filiou, M. D. \& Sandi, C. Anxiety and brain mitochondria: A bidirectional crosstalk. Trends Neurosci. 42, 573-588 (2019).

54. Pei, L. \& Wallace, D. C. Mitochondrial etiology of neuropsychiatric disorders. Biol. Psychiatry 83, 722-730 (2018).

55. Preston, G., Kirdar, F. \& Kozicz, T. The role of suboptimal mitochondrial function in vulnerability to post-traumatic stress disorder. J. Inherit. Metab. Dis. 41, 585-596 (2018).

56. Picard, M. \& McEwen, B. S. Psychological stress and mitochondria: A conceptual framework. Psychosom. Med. 80, 126-140 (2018).

57. Longo, N., Frigeni, M. \& Pasquali, M. Carnitine transport and fatty acid oxidation. Biochim. Biophys. Acta 1863, 2422-2435 (2016).

58. Houten, S. M. \& Wanders, R. J. A. A general introduction to the biochemistry of mitochondrial fatty acid beta-oxidation. J. Inherit. Metab. Dis. 33, 469-477 (2010).

59. Koren, D. \& Palladino, A. in Genetic diagnosis of endocrine disorders (eds Roy E. Weiss \& Samuel Refetoff) 66 (Academic Press, 2016).

60. Nogacka, A. M. et al. Early microbiota, antibiotics and health. Cell. Mol. Life Sci. 75, 83-91 (2018).

61. Lach, G. et al. Enduring neurobehavioral effects induced by microbiota depletion during the adolescent period. Transl. Psychiatry 10, 382. https://doi.org/10.1038/s41398-020-01073-0 (2020).

62. Desbonnet, L. et al. Gut microbiota depletion from early adolescence in mice: Implications for brain and behaviour. Brain Behav. Immun. 48, 165-173. https://doi.org/10.1016/j.bbi.2015.04.004 (2015).

63. Krolow, R., Arcego, D. M., Noschang, C., Weis, S. N. \& Dalmaz, C. Oxidative imbalance and anxiety disorders. Curr. Neuropharmacol. 12, 193-204 (2014).

64. Umathe, S. N. et al. Role of nitric oxide in obsessive-compulsive behavior and its involvement in the anti-compulsive effect of paroxetine in mice. Nitric Oxide 21, 140-147 (2009). 
65. Lyall, K. et al. The changing epidemiology of autism spectrum disorders. Annu. Rev. Public Health 38, 81-102. https://doi.org/10. 1146/annurev-publhealth-031816-044318 (2017).

66. Franke, B. et al. Live fast, die young? A review on the developmental trajectories of ADHD across the lifespan. Eur. Neuropsychopharmacol. 28, 1059-1088. https://doi.org/10.1016/j.euroneuro.2018.08.001 (2018).

\section{Acknowledgements}

We thank the bio-technicians at the Central Animal Laboratory (CDL) of the Radboud university medical center for taking excellent care of the animals. This work was made possible by the generosity of the Marriott family (TK).

\section{Author contributions}

A.J.K. and T.K. contributed to the conception of the study. A.C.T., T.L.E., A.J.K. and T.K. contributed to the design of the study. A.C.T., T.L.E., B.G., V.V. and M.v.B. contributed to the acquisition and analysis of the data. A.C.T., T.L.E., E.M., A.J.K. and T.K. contributed to drafting and critically revising the manuscript. All authors reviewed and approved the manuscript.

\section{Competing interests}

The authors declare no competing interests.

\section{Additional information}

Correspondence and requests for materials should be addressed to T.K.

Reprints and permissions information is available at www.nature.com/reprints.

Publisher's note Springer Nature remains neutral with regard to jurisdictional claims in published maps and institutional affiliations.

(c) (i) Open Access This article is licensed under a Creative Commons Attribution 4.0 International cc) License, which permits use, sharing, adaptation, distribution and reproduction in any medium or format, as long as you give appropriate credit to the original author(s) and the source, provide a link to the Creative Commons licence, and indicate if changes were made. The images or other third party material in this article are included in the article's Creative Commons licence, unless indicated otherwise in a credit line to the material. If material is not included in the article's Creative Commons licence and your intended use is not permitted by statutory regulation or exceeds the permitted use, you will need to obtain permission directly from the copyright holder. To view a copy of this licence, visit http://creativecommons.org/licenses/by/4.0/.

(C) The Author(s) 2021 